\title{
HEAVY FLAVOUR MULTIPLICITIES AT VERY HIGH ENERGIES
}

\author{
W. Furmanski $\left.{ }^{*}\right)$, R. Petronzio ${ }^{* *}$ and S. Pokorski ${ }^{* *}$ ) \\ CERN - Geneva
}

\begin{abstract}
We calculate the total multiplicity of heavy flavours in $e^{+} e^{-}$annihilation at very high energies in the framework of QCD. The problem is dominated by the soft limit of the perturbative expansion and we develop suitable techniques to resum to all orders the leading double logarithmic terms. The results indicate at very high energy a growth of multiplicity faster than any power of log. We find an interesting asymptotic factorization of energy dependence and flavour mass dependence of multiplicities.
\end{abstract}

*) On leave of absence from the Institute of Physics, Jagellonian University, Cracow.

**) On leave of absence from the Istituto di Fisica dell' Università, Roma, Italy.

***) On leave of absence from the Institute for theoretical Physics, Warsaw University.

Ref.TH. 2625-CERN

25 January 1979 


\section{INTRODUCTION}

We analyze the problem of total heavy flavour multiplicity in $\mathrm{e}^{+} \mathrm{e}^{-}$ annihilation at high energies. The problem is interesting in several aspects. On the experimental side, it is useful to have some expectations on the heavy quark content of high energy $e^{+} e^{-}$jets. The rise of multiplicity coming from gluon bremsstrahlung (converting into heavy pairs) produces a contamination to the original heavy quark content of the jet and may affect possible tests of weak decay patterns of the initial heavy quark. Futhermore, we may expect that heavy flavour multiplicity at high $Q^{2}$ depends crucially on the non-Abelian nature of the interactions. At high energies where higher order diagrams peculiar to QCD are important (see fig. 1), the, say, charm multiplicity $\mathrm{N}_{c}\left(Q^{2}\right)$ should provide important tests of the theory. Finaliy, the problem is interesting technically and goes beyond the previous perturbative calculations in QCD. On one side, due to the presence of the heavy quark mass $M_{c}$ in the problem, we believe that a perturbative approach is justified 1). On the other hand, since we are interested in the total multiplicity, we explicitly enter the infra-red domain of the theory or, by using the language of the hadron phenomenology, we investigate the rapidity plateau region of charm spectrum. Here the genuine hadron physics takes place, fixed Feynman $x$ region being only a small tail of what one really observes.

When energies get large, entering the soft region of inclusive spectra means resumming double logarithmic $\alpha^{n} l_{n}{ }^{2 n} \frac{Q^{2}}{M^{2}}$ contributions. In the previous applications of perturbative $Q C D{ }^{c}$ the infrared problem has been usually avoided by choosing specific physical problems. In particular recent attention of many authors has been concentrated on obtaining QCD predictions for the $Q^{2}$ dependence of the quark fragmentation (or structure) functions in the finite $x$ region. In the leading logarithmic approximation they are determined by the leading mass (collinear) singularities $\alpha^{n} \ell_{n}{ }^{n} \frac{Q^{2}}{M} 2^{*}$ It has been proved that the summation of the leading collinear singularities can be easily performed in any axial gauge. In such a gauge only ladder diagrams give leading contributions and one is led to a simple probablistic picture of the $Q^{2}$ evolution $\left.{ }^{2)}, 3\right)$. The extrapolation of the above results to the soft region $x \sim \frac{M^{2}}{Q^{2}}$ is not allowed since, in technical words, the neglected terms $\frac{1}{x} \operatorname{lnx}$ become as important as terms $\frac{1}{x} l_{n} \frac{Q^{2}}{M^{2}}$ leading in the collinear configuration ${ }^{*}$ ).

*) The region of low but fixed $x$ has been investigated by many authors within the single leading log approximation. See, e.g., Refs. 4-5). 
In this paper we show that with the appropriate choice of the specific axial gauge also in our problem only the ladder diagrams must be summed in the leading log approximation (ILA) and consequently we are able to find the solution for the multiplicity of heavy quark-antiquark pairs as a function of $Q^{2}$. The most important feature of this solution is the asymptotic rise of the multiplicity as

\section{$\exp \left(\sqrt{\alpha \ln Q^{2} / Q_{0}^{2}}\right)$}

which reflects the presence of the $3 \mathrm{~g}$ coupling in the theory. This growth, faster than any power of log, is a clearcut prediction, when compared with the present logarithmic growths of hadronic multiplicities. Hereafter, we specialize to the case of charm pairs but the results are obviously valid for any heavy flavour.

In the next section we discuss the problem of the right choice of gauge using as a laboratory the lowest order contribution to the charm production. Section 3 contains further arguments which allow us to select diagrams which must be resummed in our problem. In Section 4 the integral equation for the multiplicity and its solution are given. The last section is devoted to numerical estimates and to concluding remarks.

\section{IOWEST ORDER CONTRIBUTION}

In the lowest non-trivial order the contribution to the charm multiplicity is given by the diagrams in Fig. 2. The case where the observed charm quark is that originally produced by the photon (nonsinglet channel) contributes to the average multiplicity $\langle n\rangle=\sum_{n} \sigma_{n} / \sigma_{\text {tot }}$ by a trivial term

$$
\langle n\rangle^{N . S}=3 e_{c}^{2} / R
$$

where $e_{c}^{2}$ is the electric charge of charm and $R=3 \sum_{i} e_{i}^{2}$ the sum being extended to all quark flavours. In the following by multiplicity we always understand multiplicity of qā pairs. The leading contribution to the charm multiplicity $\mathrm{N}_{c}\left(\mathrm{Q}^{2}\right)$ from diagrams in Fig. 2 is proportional to $\log ^{3} \mathrm{Q}^{2} / 4 \mathrm{M}_{\mathrm{C}}^{2}$ and can be easily calculated in an arbitrary axial gauge.

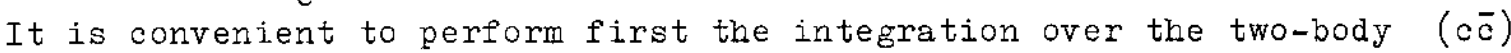
phase space and the multiplicity $\mathbb{N}_{C}\left(Q_{Q^{2}}^{2}\right)$ can then be written as follows:

$$
N_{C}\left(Q^{2}\right)=\frac{\alpha}{2 \pi} \frac{2}{3} T_{F} \int_{4 M_{C}}^{Q^{2}} \frac{d k^{2}}{k^{2}} N_{G}\left(k^{2}\right)
$$

where $\mathrm{N}_{\mathrm{G}}\left(\mathrm{k}^{2}\right)$ is formally calculated as the multiplicity of gluons with the mass $k^{2}$ summed over final polarizations and colours (where $T_{F}=\frac{1}{2}$ for $\mathrm{SU}(3)$ colour). To order $\alpha$ there are three diagrams contributing 
to $\mathbb{N}_{G}\left(k^{2}\right)$, as shown in Figs. $3 a$ and $3 b$. In a gauge defined by $n \cdot A=0$ with an arbitrary vector $n$ the leading contributions to $N_{G}\left(k^{2}\right)$ from the diagrams in Figs. $3 a$ and $3 b$ can be obtained by neglecting the gluon momentum $k$ in the numerator of the full amplitude and by keeping terms with the product $\mathrm{kn}$ in the denominators (coming from the gluon propagator

$$
d_{\mu \nu}(k)=-g_{\mu \nu}+\frac{k_{\mu} m_{\nu}+k_{\nu} m_{\mu}}{k m}+\frac{m^{2} k_{\mu} k_{\nu}}{(k m)^{2}}
$$

in addition to the quark propagators. As will be shown below by explicit calculation those terms give also leading contribution after integration.

The leading contribution of diagrams in Fig. $3 a$ reads:

$N_{G}^{(1)}\left(k^{2}\right)=\frac{1}{\sigma_{0}} \int \frac{d \sigma_{0}\left(p_{1}, p_{2}\right)}{d R_{2}\left(p_{1}, p_{2}\right)} 2 C_{F} g^{2}\left[\frac{\left(p_{1} n\right)}{\left(p_{1} k\right)(k m)}+\frac{\left(p_{2} n\right)}{\left(p_{2} k\right)(k m)}\right] d R_{3}\left(p_{1}, p_{2}, k\right)^{(2.2)}$

and that of diagrams in Fig. $3 \mathrm{~b}$ is

$N_{G}^{(B)}\left(k^{2}\right)=\frac{1}{\sigma_{0}} \int \frac{d \sigma_{0}\left(p_{1}, p_{2}\right)}{d R_{2}\left(p_{1}, p_{2}\right)} \frac{2 C_{F} g^{2}}{\left(p_{1} k\right)\left(p_{2} k\right)}\left[p_{1} p_{2}-\frac{\left(p_{1} n\right)\left(p_{2} k\right)}{(k m)}-\frac{\left(p_{2} n\right)\left(p_{1} k\right)}{(k m)}\right] d R_{3}\left(p_{1}, p_{2}, k\right)$

where $\mathrm{C}_{f}=\frac{4}{3}, \mathrm{~g}$ is the strong coupling constant and $\sigma_{0}$ is the Born cross-section:

$$
\frac{d \sigma_{0}\left(p_{1}, p_{2}\right)}{d R_{2}\left(p_{1}, p_{2}\right)}=R \frac{1}{2 Q^{2}}\left(\frac{e^{2}}{Q^{2}}\right)^{2} \frac{1}{4} \operatorname{Tr}\left[\not_{1} \gamma^{\mu} \chi_{2} \gamma^{\nu}\right] \operatorname{Tr}\left[p_{1} \gamma \gamma^{\mu} \not_{2} \gamma^{\nu}\right]
$$

with $R=3 \Sigma_{i} e_{i}^{2}, \quad e_{i}$ being the $i^{\text {th }}$ quark charge in units of proton charge e. $R_{2}, R_{3}$ denote the two-and three-body phase space respectively.

We observe first that in the collinear configuration $k=x_{i}$ ( $i=1$ or 2) the square bracket in $\mathrm{Eq}$. (2.3) vanishes and the full result is given by the ladder diagrams of $\mathrm{Fig}$. $3 \mathrm{a}$. We can ask whether the same occurs in the soft domain of the phase space where the dominant part of the multiplicity comes from.

The answer is negative and this can be shown by the explicit 
integration of the ladder diagram contribution (2.2). The integration over $d R_{3}$ can be replaced by two succesive two-body phase-space integrations:

$$
N_{G}^{(A)}\left(k^{2}\right)=\frac{1}{\sigma_{0}} \int d \sigma_{0}\left(p, p_{2}\right) \int d R_{2}\left(k, p_{1}\right) 2 C_{F} \frac{q^{2}}{\pi}\left(\frac{p_{m} m}{k n}\right)+(1 \leftrightarrow 2)
$$

where $\mathrm{p}=\mathrm{p}_{1}+\mathrm{k}$.

The invariant integration over $\mathrm{dR}_{2}$ can be conveniently done in the $p$ rest frame:

$$
N_{G}^{(A)}\left(k^{2}\right)=\frac{1}{\sigma_{0}} \int d \sigma_{0}\left(p, p_{2}\right) \frac{\alpha}{2 \pi} 2 C_{F} \int \frac{d \Omega}{4 \pi}\left(\frac{p_{1} m}{k m}\right)+(1 \leftrightarrow 2) .
$$

Integrating over the solid angle we get the logarithmic contribution when $k|| n$ :

$$
\int \frac{d \Omega}{4 \pi}\left(\frac{p n}{k n}\right)=\frac{1}{\sqrt{1-\tau}} \ln \frac{x_{M A x}}{x_{M I N}}
$$

where

$$
\underset{\text { (niN) }}{x_{\max }}=\frac{1}{2}\left[1+\frac{k^{2}}{p^{2}} \pm \sqrt{1-\tau}\left(1-\frac{k^{2}}{p^{2}}\right)\right]
$$

and $T=p^{2} n^{2} /(p n)^{2}$.

For an arbitrary $n$ the result is gauge dependent which indicates that the interference diagram cannot be neglected in the soft limit in an arbitrary non-covariant gauge. Indeed, as seen from the formulae (2.2) and $(2.3)$ the gauge dependent terms of interference diagrams cancel completely the contribution of the diagram A. The remaining, explicitly $n$ independent term (being exactly the expression for the interference diagram in the Feynman gauge) gives after integration

$$
N_{G}\left(k^{2}\right)=\frac{\alpha}{2 \pi} C_{F} \operatorname{lu}^{2} \frac{Q^{2}}{k^{2}}
$$

Thus, apparently an arbitrary axial gauge seems rather to complicate, instead of simplify, the description of the soft radiation. The situation 
changes radically after further specification of the gauge. Returning to the formula (2.3) we observe that it vanishes identically also in the soft limit if we use a planar gauge defined by ${ }^{5}$ )

\section{$m=A p_{1}+B p_{2}$}

with $A$ and $B$ arbitrary constants.

In this class of gauges (and only in this class) the ladder term alone gives the full leading contribution to the multiplicity. A simple calculation shows that the gauge (i.e. A and B) dependence cancel after adding both terms of Eq. (2.2) and we get the correct result (2.5).

A particularly interesting choice of the planar gauge, which will be used in this paper, is that with $A$ (or B) equal zero. Let us fix for definiteness $n=p_{2}$. As seen from $E q$. (2.2), in this case the whole radiation comes from the quark line with momentum $p_{1}$. Since $n^{2}=0$ we may put $\tau=0$ in $\mathrm{Eq} .(2.4)$ and taking into account only one of the two diagrams of $\mathrm{Fig}$. 3 a (only one leg radiates) we get again the result $(2.5)$.

One can easily demonstrate that the condition $n=p_{2}$ eliminates soft radiation from the quark line $\mathrm{p}_{2}$ to any order. By anticommuting the $\gamma$ matrices in the ggG vertex $\mathrm{J}^{\mu}\left(\mathrm{p}_{2}\right) \mathrm{A}_{\mu}(\mathrm{k})$ we see that in the soft limit the current $J^{\mu}\left(p_{2}\right)$ is classical: $J^{\mu}\left(p_{2}\right)=2 p_{2}^{\mu}$. Because of the gauge condition $\mathrm{nA}=0$ and our choice $\mathrm{n}=\mathrm{p}_{2}$ the vertex vanishes. The fact that in this collinear gauge the radiation comes only from one leg does not contradict physical intuition. In the soft limit this mechanism cannot be distinguished from the emission from both legs. The collinear gauge $\mathrm{n}=\mathrm{p}_{2}$ we have chosen presents an additional technical advantage in the extension of the calculation to higher orders due to the fact that $n$ is now light-like. For $n^{2} \neq 0$ the parameter $\tau=p^{2} n^{2} /(p n)^{2}$ couples the angular integrations in different cells of the ladder, making the calculation in higher orders hopelessly complicated. Only for $\mathrm{n}^{2}=0$ we may integrate independently cell by cell along the ladder, which enables us to solve the problem for multiplicity.

The logarithmic contributions from the gauge denominators $1 / \mathrm{kn}$, looking very artificial when derived in the rest frame of the radiating fermion have for the collinear gauge a very simple interpretation in the infinite momentum frame, (IMF).

Let us turn back to the diagram $A$ and parametrize the momenta as follows ${ }^{6)}$ : 


$$
\begin{aligned}
& p=\left(p_{0}, \vec{p}_{T}, p_{3}\right)=\left(p+\frac{p^{2}}{4 M}, \overrightarrow{0}, p-\frac{p^{2}}{4 M}\right), \\
& k=\left(x p+\frac{k^{2}+k_{T}^{2}}{4 x p}, \vec{k}_{T}, x p-\frac{k^{2}+k_{T}^{2}}{4 \times p}\right), \\
& n=\left(\frac{p \eta}{2 p}, 0,-\frac{p \eta}{2 p}\right),
\end{aligned}
$$

so that $\frac{n k}{p n}=x$. We may interpret the Eqs. (2.6) as follows: since $P$ is an arbitrary boost parameter defining the frame (invariant quantities are $P$ independent) we may start, e.g., from the $p$ rest frame $\left(4 P^{2}=p^{2}\right)$ and make an infinite boost $(P \rightarrow \infty)$ in the direction opposite to $\vec{n}$. Now the gauge denominators $\frac{\mathrm{kn}}{\mathrm{pn}}$ can be interpreted as the momentum fraction of the radiating particle carried by the gluon in the IMF.

A simple calculation shows that

$$
\left[\int \frac{d \Omega}{4 \pi}\left(\frac{p m}{k n}\right)\right]_{p C M S}=\left[\int_{k^{2} / p^{2}}^{1} \frac{d x}{x}\right]_{p I M F}
$$

so that the logarithmic divergences coming from gauge denominators get the interpretation of the usual soft divergences in the IMF.

We complete this section giving the complete expression (up to constant terms) for the charm multiplicity in the lowest order. It reads:

$N_{c}\left(Q^{2}\right)=\frac{\alpha^{2}}{9 \pi^{2}}\left[\frac{1}{3} \ln ^{3} \frac{Q^{2}}{4 M_{c}^{2}}-\frac{3}{2} \ln ^{2} \frac{Q^{2}}{4 H_{c}^{2}}+\left(6-\frac{\pi^{2}}{3}\right) \ln \frac{Q^{2}}{4 M_{c}^{2}}\right]$.

Eq. (2.7) is meaningful provided the next order correction can be neglected: $\alpha \ln ^{2}\left(Q^{2} / 4 M_{c}^{2}\right) \ll 1$. When this condition is not fulfilled higher order terms are important.

\section{SELECTION OF LEADING DIAGRAMS}

In this section we show that in the gauge $\mathrm{n}=\mathrm{p}_{2}$ the maximal logarithmic contribution at any order can be obtained only from the gluon ladder, whereas crossed ladders as well as those containing the the fermion loops are down at least by one power of log. 
The last statement is obvious: since the gluon $\rightarrow \mathrm{q} \bar{q}$ vertex is hard, we always gain one power of log by replacing the quark loop by the corresponding gluon 10op. (The four-gluon vertices can be neglected for the same reason).

Thus the general structure of the leading diagrams contributing to $N_{c}\left(Q^{2}\right)$ in the gauge $n=p_{2}$ is as in Fig. 4, with the hatched blob containing only gluons. Let us start with the diagrams without crossed gluon lines attached to the two external fermions. Then, integrating over final charm pair as in section 2, we may limit the discussion to the diagrams contributing to the gluon multiplicity, according to the formula (2.1). (See Fig. 5). We adopt the convention that the integration $\mathrm{dk}^{2} / \mathrm{k}^{2}$ from Eq. (2.1) is associated with the upper line $k$ in the diagrams in Fig. 5, i.e., the Feynman rule for the line $k$ reads

$$
\frac{i d_{\mu \nu}\left(k^{2}\right)}{k^{2}} \frac{d^{4} k}{(2 \pi)^{4}} \theta\left(k^{2}-4 M_{c}^{2}\right) \text {. }
$$

Let us consider the diagrams $A$ and $B$ in Fig. 5. As will be shown in the following, the diagram $A$ gives as the leading contribution $\alpha^{2} \ln ^{4}\left(\mathrm{p}^{2} / \mathrm{M}_{\mathrm{c}}^{2}\right)$. Now, since there are only three propagators in the diagram $B$, one of the gluon lines has to contribute two powers of log in order to make the diagram $B$ leading. It means that the leading term has to come from the part

$$
\frac{k_{\mu} m_{\nu}+k_{\nu} n_{\mu}}{k^{2}(k m)}
$$

in the propagator. For definiteness, let us consider the part of a diagram depicted in Fig. 6 with $p_{1}^{2}=p_{2}^{2}=0$. Since the leading contribution is obtained when $k|| n$ and $k^{2}=0$, in the chosen lightlike gauge $n^{2}=0$ we may write in the numerator $k=\lambda_{n}$ where

$$
\lambda=\frac{k \cdot k_{1}}{m \cdot k_{1}}=\frac{k k_{2}}{m k_{2}}=\sqrt{\left(\frac{k k_{1}}{n k_{1}}\right)\left(\frac{k k_{2}}{m k_{2}}\right)} \text {. }
$$

Due to the identity $n^{\mu} d_{\mu \nu} \equiv 0$ there is only one allowed flow of Lorentz indices through the vertices (dotted lines in Fig. 6) and we get finally the factor

$$
\frac{2 k_{1}^{2} \sqrt{x_{1}}}{\sqrt{k_{1}^{2}+k^{2}}} \frac{1}{k^{2} \times} \frac{2 k_{2}^{2} \sqrt{x_{2}}}{\sqrt{k_{2}^{2}+k^{2}}}
$$

for the line $k$ with $x_{i}=\frac{k_{i} n}{p n}, i=1,2, \quad x=\frac{k n}{p n}, \quad p$ being defined in Fig.5. 
The logarithmic contribution from $\mathrm{dk}^{2}$ integration comes only from the region $k_{2}^{2} \gg k^{2}$ and $k_{1}{ }^{2} \gg k^{2}$; because of kinematics these conditions are satisfied only if the line $k$ is the upper gluon line in the diagram $B$. But now the factors

\section{$\sqrt{k_{1}^{2} x_{1}}, \sqrt{k_{2}^{2} x_{2}}$}

kill the logarithmic contributions coming from the two simple poles in the independent integrations over $\mathrm{k}_{1}^{2}, \mathrm{k}_{2}^{2}$. This makes diagram $B$ non leading. The situation is entirely different for the ladder diagram: here the damping of order $k_{1}^{2}$ which occurs when $k_{1}=k_{2}$ in Eq. (3.1)! is compensated by a corresponding double pole in the $k_{1}^{2}$ integration.

The physical meaning of the damping factors in the formula (3.1) is the following: by taking the part of $d_{\mu \nu}(k)$ parallel to $n$ and imposing the condition $k|| n, k^{2}:=0$, we force the gluon $k$ to be longitudinal and on-shell. Since it cannot be produced in a physical process $k_{1} \rightarrow k_{1}+p_{1}, k_{2} \rightarrow k+p_{2}$, we get factors $\sqrt{k_{1}^{2}}, \sqrt{k_{2}^{2}}$, assuring that the line $k$ is an internal line of the diagram. Factors $V x_{1}, V x_{2}$ are of kinematical origin. E.g., $x_{1}$ is small when $k_{1}|| k$, i.e., in a kinematically forbidden configuration when we try to produce two massless collinear particles $p_{1}$, $k$ from the massive particle $k_{1}$. The argument presented above works in any order: we consider first diagrams without virtual corrections. An arbitrary $n^{\text {th }}$ order interference diagram contributing to the hatched blob of Fig. 5 has $2 n-1$ propagators: in order to build up the leading $\alpha^{n} l_{n}{ }^{2 n} p^{2} / m^{2}$ contribution we need at least one gluon to be longitudingl and $\mathrm{Eq}$. (3.1) can be used. Since in interference diagrams all internal momenta are in general different and may overlap only in a negligible region of phase space, the square root factors $\sqrt{k_{1}^{2} x_{1}}, V_{k_{2}}^{2} x_{2}$ will always kill the simple poles of corresponding independent integrations. As a consequence all interference diagrams are non leading. For ladder diagrams the mechanism described before for a simple case works cell by cell giving rise to a $\alpha^{n} l_{n} 2 p^{2} / \mathbb{M}^{2}$ contribution. In a similar way one may eliminate diagrams with the crossed gluons attached to fermions (Fig. 7). Again, by counting the propagators labeled by different momenta and comparing with the ladder diagram we conclude that one gluon has to be longitudinal. It cannot be coupled to charm because of the current conservation, whereas if it is attached to the initial quark, the formula (3.1) is valid (after anticommuting the $\gamma$ matrices sandwiching the gluon vertex).

The counting argument used extensively before breaks down for fermion 
loops. Since we lose one log when $q \bar{q}$ pair is produced, the number of logs in the ladder may be equal to the number of propagators in the interference diagram and we may get leading terms without longitudinal gluons. Since, contrary to internal fermion loops, we cannot avoid the initial "non-leading" quark loop, the choice of the planar gauge is essential. In order to illustrate the relevance of the collinear gauge in retaining the ladder structure in the soft limit we may take for comparison leading diagrams in the Feynman gauge. Here, all leading logs have to be constructed from usual propagators and the line $p_{2}$ plays an essential role (Fig. B). Factors $1 / k_{1} p_{2}$, appearing as the gauge denominators of the ladder gluons in the planar gauge, are generated in the Feynman gauge by explicit coupling of the soft radiation to the line $p_{2}$.

Virtual diagrams do not give rise to double logs since their soft divergences cancel with the corresponding real undetected emission. Thus our selection of diagrams in the double log approximation is completed. It is worth remembering here that in the double log approximation one neglects all terms $\alpha\left(\mu^{2}\right) \log k^{2}$ as compared to terms $\alpha\left(\mu^{2}\right) \log ^{2} k^{2}$ for $4 \mathbb{H}_{c}^{2} \leq k^{2} \leq Q^{2}$ where $\mu^{2}$ is a renormalization point. However, among all the single logarithmic terms there is an important subset which cannot be neglected, namely the terms which provide the renormalization of the strong coupling constant. As in all renormalization group improved calculations these terms can be easily resummed by introducing at each cell the running coupling constant renormalized at the invariant momentum $\mathrm{k}^{2}$ flowing in the cell:

$$
\alpha\left(k^{2}\right)=\frac{\alpha\left(Q_{0}^{2}\right)}{1+\frac{\alpha\left(Q_{0}^{2}\right)}{\pi} b \ln \frac{k^{2}}{Q_{0}^{2}}} \quad, b=\frac{33-2 N_{F}}{12} .
$$

As will be seen from the explicit calculation in the next section the multiplicative effect of the running coupling constant modifies the asymptotic form of the solution obtained in the double logarithmic approximation (with fixed coupling $\alpha\left(k^{2}\right)=\alpha\left(\mu^{2}\right)$ ). As seen from (3.2) the effect is important for high $Q^{2}$, namely when the condition $\alpha\left(\mu^{2}\right)\left|\ell_{n k}{ }^{2} / \mu^{2}\right| \ll \frac{1}{2}$ cennot be satisfied for all values of $k^{2}$ in the range $4 \mathbb{M}_{\mathrm{C}}^{2} \leq \mathrm{k}^{2} \leq Q^{\overline{2}}$ (by any choice of renormalization point $\mu^{2}$ ). Some arguments for the consistency of including only the considered subset of single log terms will be given in the next section. 


\section{INTEGRAL EQUATION FOR THE MULTIPLICITY}

In this section we will sum up the gluon ladder. which was found before to give the leading contribution to the charm multiplicity.

The Bethe-Salpeter equation (BSE) for the gluon ladder is presented in Fig. 9 where the diagram on the LHS (with non-amputated fermion legs and with fixed momentum p) will be denoted as

$$
\text { LHS }=\hat{n}_{G}(p, k) \frac{\not p}{p^{2}} .
$$

In the leading log approximation, the quantity $\hat{n}_{G}$ (in general a spinor matrix) is simply a scalar function of $\mathrm{p}^{2}, \mathrm{k}^{2}$.

It ean be interpreted as the multiplicity of gluons with mass $\mathrm{k}^{2}$ inside the quark with the mass $p^{2}$ and is related to $\mathbb{N}_{G}\left(k^{2}\right)$ as follows:

$$
N_{G}\left(k^{2}\right)=\int_{k^{2}}^{Q^{2}} \frac{d p^{2}}{p^{2}} m_{G}\left(p^{2}, k^{2}\right) .
$$

We will calculate $n_{G}\left(p^{2}, k^{2}\right)$ in the infinite momentum frame, parametrizing the momenta as follows:

$$
\begin{aligned}
& p=\left(p+\frac{p^{2}}{4 p}, 0, p-\frac{p^{2}}{4 p}\right), \\
& n=\left(\frac{p n}{2 p}, 0,-\frac{p n}{2 p}\right), \\
& k=\left(x p+\frac{k^{2}+k_{T}^{2}}{4 x p}, \vec{k}_{T}, x p-\frac{k^{2}+k_{T}^{2}}{4 x p}\right), \\
& l=\left(y p+\frac{l^{2}+l_{T}^{2}}{4 y p}, \vec{\ell}_{T}, y p-\frac{l^{2}+l_{T}^{2}}{4 y p}\right), \\
& x=\frac{k n}{p m}, y=\frac{\ell n}{p m} .
\end{aligned}
$$

The invariant integration is:

$$
d^{4} k=d k^{2} \frac{d x}{2|x|} d^{2} k T .
$$

To extract the maximal logarithmic contribution from the line $k$ on the RHS of BSE in Fig. 9, we use formula (3.1). Performing the integration over $d^{2} \vec{k}_{q}$ in the first term on the RHS of our BSE with: 
$-11-$

$$
\delta\left((p-k)^{2}\right)=\delta\left((1-x)\left(p^{2}-\frac{k^{2}}{x}\right)-\frac{k_{T}^{2}}{x}\right)
$$

we get from the conditions $k_{T}^{2}>0,(p-k)_{0}>0$

$$
k^{2} / p^{2}<x<1 \text {. }
$$

In the second term on the RHS of our equation we introduce relative coordinates $\bar{z}=\mathrm{x} / \mathrm{y}, \quad \vec{r}_{\mathrm{T}}=\overrightarrow{\mathrm{k}}_{\mathrm{T}}-\mathrm{z}_{\mathrm{l}} \overrightarrow{\mathrm{T}}_{\mathrm{T}}$ and integrate over $\mathrm{d}^{2} \vec{r}_{\mathrm{T}}$ with

$$
\delta\left((l-k)^{2}\right)=\delta\left((1-z)\left(l^{2}-\frac{k^{2}}{z}\right)-\frac{\pi_{1}^{2}}{z}\right)
$$

which gives

$$
k^{2} / l^{2}<z<1
$$

Summing over colours we get finally

$$
m_{G}\left(p^{2}, k^{2}\right)=\frac{\alpha\left(p^{2}\right)}{2 \pi} \int_{k^{2} / p^{2}}^{1} d x \frac{2 C_{F}}{x}+\int_{k^{2}}^{p^{2}} \frac{d l^{2}}{l^{2}} \frac{\alpha\left(l^{2}\right)}{2 \pi} \int_{k^{2} / l^{2}}^{1} d z \frac{2 C_{G}}{Z} m_{G}\left(p^{2}, l^{2}\right) .
$$

We will solve equation (4.2), using the following set of variables and parameters:

$$
\begin{aligned}
& \alpha\left(k^{2}\right)=\frac{\alpha\left(Q_{0}^{2}\right)}{1+\frac{\alpha\left(Q_{0}^{2}\right)}{\pi} b \ln \frac{K^{2}}{Q_{0}^{2}}}, b=\frac{33-2 N_{F}}{12}, \\
& y=\frac{\pi}{b \alpha\left(k^{2}\right)}, Y=\frac{\pi}{b \alpha\left(p^{2}\right)}, \eta=\frac{\pi}{b \alpha\left(l^{2}\right)} \text {, } \\
& a_{G}=\frac{4 C_{G}}{b}, a_{F}=\frac{4 C_{F}}{b}, a_{C}=\frac{4}{b} \frac{T_{F}}{3} \text {. }
\end{aligned}
$$

Equation (4.2) may be rewritten as follows:

$$
m_{G}(Y, y)=\frac{1}{4} a_{F} \frac{Y-y}{y}+\frac{1}{4} a_{G} \int_{y}^{Y} d \eta \frac{\eta-y}{\eta} m_{G}(Y, \eta) .
$$


Differentiating twice over $y$ we get

$$
y \frac{d^{2}}{d y^{2}} n_{G}(Y, y)=\frac{1}{4} a_{G} n_{G}(Y, Y)
$$

with boundary conditions

$$
\begin{aligned}
& n_{G}(Y, Y)=0, \\
& \left.\frac{d}{d y} m_{G}(Y, y)\right|_{y=Y}=-\frac{a_{F}}{4 I} .
\end{aligned}
$$

By substitution $Z=V_{a_{G}} y, \quad \hat{n}_{G}(Y, y)=Z w(Z)$ we reduce $\mathrm{Eq} \cdot(4 \cdot 3)$ to the Bessel equation:

$$
z^{2} \omega^{\prime \prime}(z)+z \omega^{\prime}(z)-\left(z^{2}+1\right) \omega(z)=0
$$

Two Iinearly independent solutions of (4.4) are the modified Bessel functions $I_{1}(z), K_{1}(z)$. Imposing the boundary conditions we get finally

$$
m_{G}(Y, y)=\frac{1}{2} a_{F} \frac{z}{Z_{0}}\left[I_{1}\left(z_{0}\right) K_{1}(z)-I_{1}(z) K_{1}\left(z_{0}\right)\right]
$$

where

$$
z=\sqrt{a_{G} y}, z_{0}=\sqrt{a_{G} Y}
$$

We perform the integrations as in Eqs. (4.1) and (2.1) replacing in the latter $\alpha$ with $\alpha\left(k^{2}\right)$ inside the integral and we get the final formula for the charm multiplicity:

$$
\begin{aligned}
N_{C}\left(Q^{2}\right) & =\frac{Q_{F} a_{C}}{2 Q_{G}}\left[I_{0}\left(\sqrt{a_{G}\left[\frac{\pi}{b \alpha\left(Q_{0}^{2}\right)}+\ln \frac{Q^{2}}{Q_{0}^{2}}\right]}\right) K_{0}\left(\sqrt{a_{G}\left[\frac{\pi}{b \alpha\left(Q_{0}^{2}\right)}+\ln \frac{4 M_{0}^{2}}{Q_{0}^{2}}\right]}\right)-\right. \\
& -I_{0}\left(\sqrt{a_{G}\left[\frac{\pi}{b \alpha\left(Q_{0}^{2}\right)}+\ln \frac{4 M_{0}^{2}}{Q_{0}^{2}}\right]}\right) K_{0}\left(\sqrt{a_{G}\left[\frac{\pi}{b \alpha\left(Q_{0}^{2}\right)}+\ln \frac{Q^{2}}{Q_{0}^{2}}\right]}\right) \\
& \left.-\frac{1}{2} \ln \left(\frac{\frac{\pi}{b \alpha\left(Q_{0}^{2}\right)}+\ln \frac{Q^{2}}{Q_{0}^{2}}}{\frac{\pi}{b \alpha\left(Q_{0}^{2}\right)}+\ln \frac{4 H_{2}^{2}}{Q_{0}^{2}}}\right)\right] .
\end{aligned}
$$


When $Q^{2} \rightarrow \infty$ only the first term in (4.5) contributes and, using the asymptotic formula for $I_{0}$ we get

$$
N_{C}\left(Q^{2}\right) \underset{Q^{2} \rightarrow \infty}{\sim} \frac{Q_{F} a_{C}}{2 a_{G}}\left(4 \pi^{2} a_{G} \ln \frac{Q^{2}}{Q_{0}^{2}}\right)^{-\frac{1}{4}} e^{\sqrt{Q_{G} \ln \frac{Q^{2}}{Q_{0}^{2}}} K_{0}\left(\sqrt{Q_{G} \ln \frac{4 M_{T}^{2}}{Q_{0}^{2}}}\right)(4.6)}
$$

From formula (4.5) we can also obtain the solution corresponding to the fixed coupling approximation $\left(\alpha\left(\mathrm{k}^{2}\right) \equiv \alpha\left(\mathrm{Q}_{0}^{2}\right)\right)$ by taking the limit $\mathrm{b} \rightarrow 0$. In this case we get

$$
N_{C}\left(Q^{2}\right)=\frac{C_{F} T_{F}}{6 C_{G}^{2}} \sqrt{C_{G} \frac{\alpha\left(Q_{Q}^{2}\right)}{\pi}}\left[\left(\frac{Q^{2}}{4 H_{C}^{2}}\right)^{\sqrt{C_{G} \frac{\alpha\left(Q^{2}\right)}{\pi}}}-\left(\frac{4 H_{C}^{2}}{Q^{2}}\right) \sqrt{C_{G} \frac{\alpha\left(Q_{Q}^{2}\right)}{\pi}}\right] .
$$

The comparison of formulae $(4.6)$ and (4.7) demonstrates the effect of the running coupling constant on the asymptotic form of the solution.

Finally, we would like to comment on the contribution of other single log terms. We can easily control constant terms in the AltarelliParisi function: ( $2 \mathrm{C}_{G} / \mathrm{x}+$ const instead of $2 \mathrm{C}_{G} / \mathrm{x}$ in Eq. (4.2)). The only effect of their inclusion is the change of the index of the Bessel functions in $\mathrm{Eq} .(4.5)$ and additive log log correction to their arguments. Consequentiy, the leading asymptotic term

remains unchanged.

$$
\exp \left(\sqrt{Q_{G} \ln Q^{2} / Q_{1}^{2}}\right)
$$

This is an argument in favour of the self-consistency of our renormalization group improved sumation of double logs.

\section{NUMERICAL ESTIMATES AND CONCLUDING REMARRS}

In the previous section we have derived an expression for heavy flavour multiplicities valid to double log accuracy. In Fig. 10 we plot the dependence of the multiplicity $\mathrm{N}_{c}\left(Q^{2}\right)$ on $Q^{2}$ in the case of charm. To the plotted result one should add the non-singlet constant contribution which for four flavours reads $\langle\mathrm{n}\rangle_{\mathrm{c}}^{\mathrm{NS}}=0.4$. We have taken $4 \mathrm{M}_{\mathrm{c}}^{2}=9 \mathrm{GeV}^{2}$; our absolute normalization is affected by the bound state 
dynamics resulting in a smearing of charm threshold for real hadrons. In order to have some crude estimate of this effect we have varied the value of $4 \mathrm{M}_{\mathrm{c}}^{2}$ from 9 to $20 \mathrm{GeV}^{2}$, getting a decrease of the result by a factor 1.7. The full line represents the prediction given by Eq. (4.5) with the term $\pi / b \alpha\left(Q_{0}^{2}\right)$ neglected and $Q_{n}=A=0.5 \mathrm{GeV}$ (it corresponds to the parametrization $\alpha\left(\mathrm{k}^{2}\right) / \pi=1 /\left(\mathrm{b}^{\ell} \mathrm{nk}^{2} / \mathrm{h}^{2}\right)$; the dashed line refers to the lowest order calculation, given in. Eq. (2.7) with running coupling constant. The choice between $\alpha\left(Q^{2}\right)$ and $\alpha\left(4 \mathbb{M}_{c}^{2}\right)$ is rather arbitrary to the lowest order: however, for comparison with the result of Eq. (4.5) at very high energies, it seems to be more reasonable to use running coupling constant. One observes a sizable difference as expected on the basis of the asymptotic behaviour given in $\mathrm{Eq}$. (4.6). It predicts a growth of multiplicity faster than any power of $\ell_{n}\left(Q^{2} / M_{c}^{2}\right)$ - a rather unusual effect in the hadron world where, at least at present energies, the multiplicities grow logarithmically. In the asymptotic region there is a factorization of the $Q^{2}$ and $\mathbb{M}^{2}$ dependence, as in the collinear single log processes. This leads us to a suggestive phenomenological application based on the interpretation of the mass $M$ as the invariant mass of a hadronic cluster with an assigned mutiplicity: the growth of total hadron multiplicity should then be proportional to the growth of number of clusters of mass $\mathbb{M}$ as given by Eq. (4.5). As a final remark we want to note that, owing to the validity of the probabilistic scheme with an appropriate choice of the gauge, the method we used for calculating total multiplicities ean be easily extended to the calculation of inclusive spectra ${ }^{7)}$.

\section{ACKNOWLEDGEMENTS}

This work was begun in collaboration with Mr. Tomasz R. Taylor from Warsaw University to whom we are grateful for his contribution to the lowest order calculation. We are grateful also to A. De Rujula, J. Prentki and $C$. Sachrajda for several interesting discussions. 


\section{REFERENCES}

1) H. Georgi and H.D. Politzer, Nucl. Phys. B136 (1978) 445,

H. Fritsch and K.H. Streng, Phys. Lett. 78B (1978) 447.

2) For a comprehensive list of references, see e.g. R.K. Ellis, H. Georgi, M. Nachacek, H.D. Politzer and G.G. Ross, CALT 68-684,

D. Amati, R. Petronzio and G. Veneziano, Nucl. Phys. B140 (1978) 54.

3) G. Parisi, Proc. 11th Rencontre de Moriond, 1976 (ed. J. Tran Thanh Van),

G. Altarelli and G. Parisi, Nucl. Phys. B126 (1977) 298.

4) A. De Rujula, S.L. Glashow, H.D. Politzer, S.B. Treiman, F. Wilczek and A. Zee, Phys Rev. D10 (1974) 1649.

5) V.N. Gribov and I. N. Lipatov, Sov. J. Nucl. Phys. 15 (1972) 438, 675, Y.I. Dokshitser, D.I. D'yakonov and S.I. Troyan, "Hard Semi-Inclusive Processes in QCD", Leningrad Preprint and Proceedings of the 18 th Leningrad Winter School (1978), available in English as SIAC Trans. 183.

6) S. Brodsky, F. Close and J. Gunion, Phys. Rev. D8 (1973) 3679.

7) We have been informed that the multiplicity problem in QCD is also considered by A. Basetto, M. Ciafaloni, G. Marchesini and by K. Konishi. 


\section{Figure captions}

Fig. 1 : An example of the diagram giving the leading contribution to the charm multiplicity at high energies.

Fig. 2 : Diagrams contributing to the lowest (non-trivial) order.

Fig. 3 : Diagrams for the virtual gluon multiplicity (see text) in the lowest order.

Fig. 4 : General structure of the leading diagrams in the soft limit: the hatched blob contains only gluons.

Fig. 5 : General structure of the leading diagrams for the virtual gluon multiplicity in the collinear gauge $\left(n=p_{2}\right)$.

Fig. 6 : Lorentz structure of the $3 g$ vertices to which the longitudinal Gluon $\left(k|| n, k^{2}=0\right.$ ) is coupled (the dots indicate the flow of Lorentz indices).

Fig. 7 : An example of (non-leading) diagrams with the crossed gluon lines attached to fermions.

Fig. 8 : The comparison of the leading diagrams in the collinear gauge and in the Feynman gauge. The dots indicate where the denominators $1 / \mathrm{kn}$ came from in both gauges.

Fig. 9 : Bethe-Salpeter equation for the gluon multiplicity.

Fig.10 : Numerical estimates: the solid line represents the prediction given by Eq. (4.5), the dashed line is the lowest order result $|\mathrm{Eq} .(2.7)|$ with the running coupling constant. 


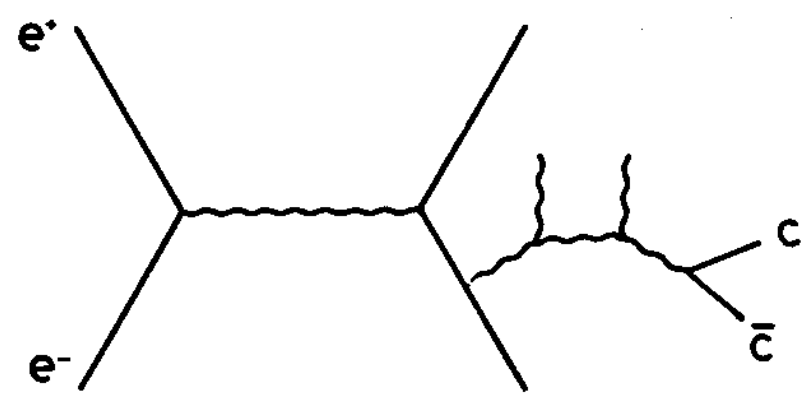

FIG.1
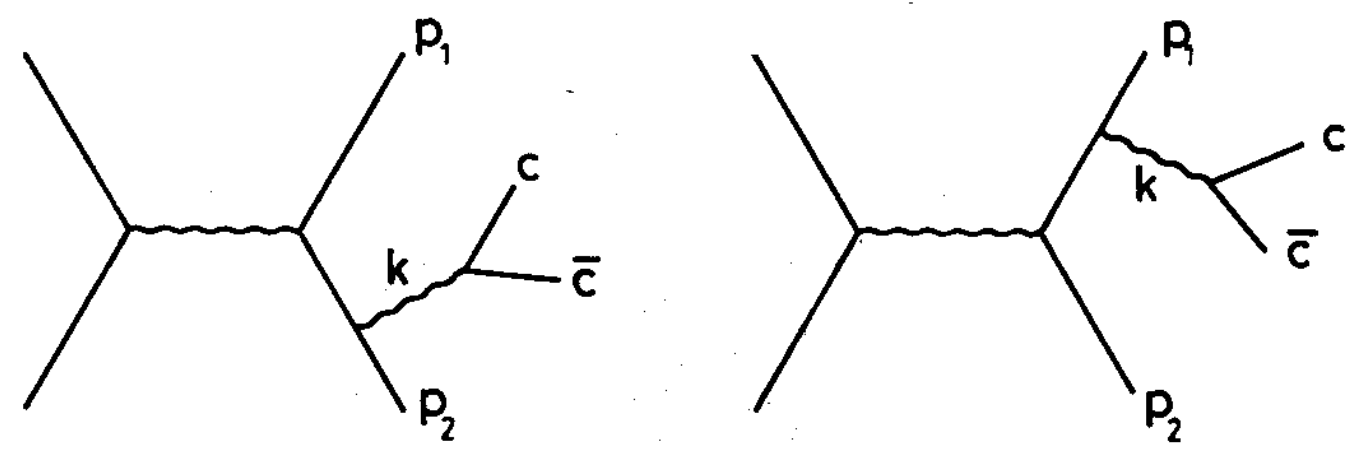

FIG.2 


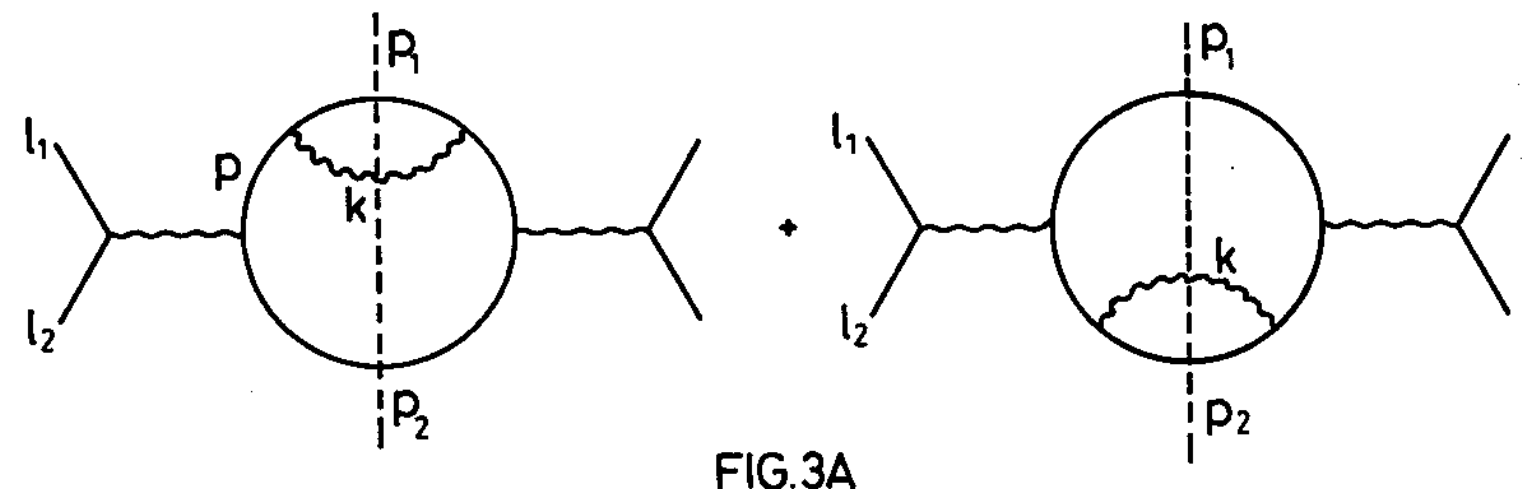

FIG.3A

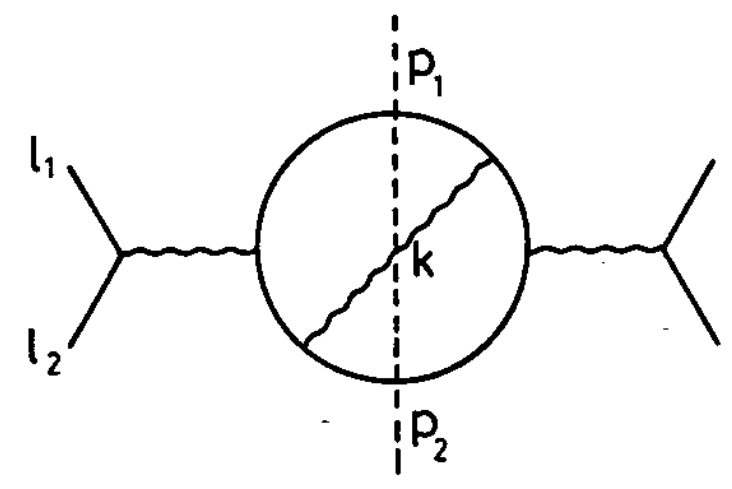

FIG. 3B

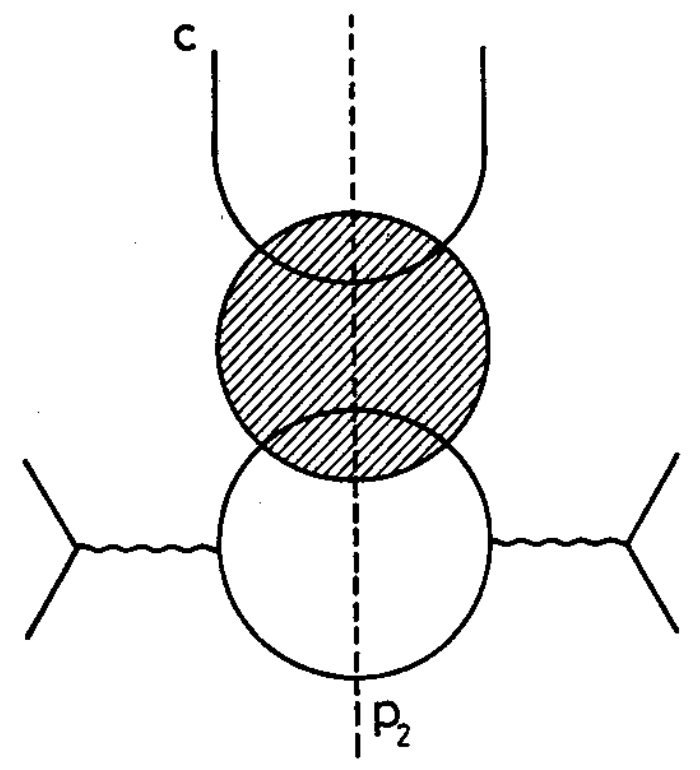

FIG. 4 

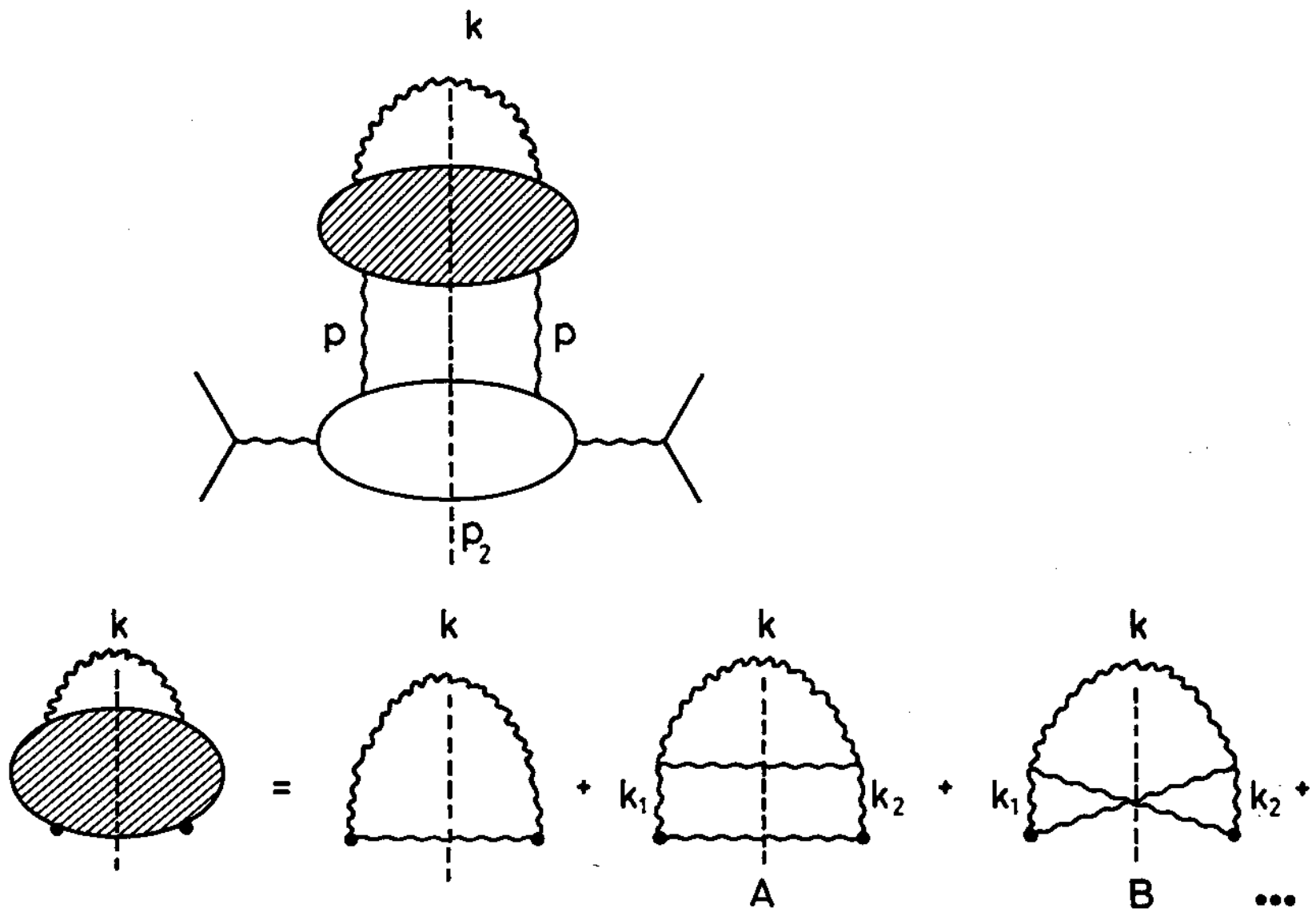

FIG. 5

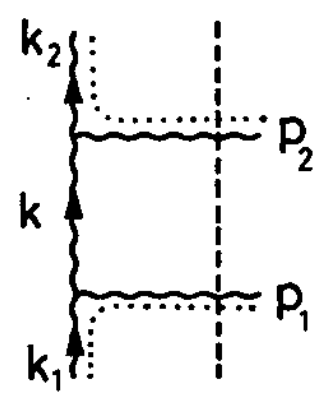

FIG. 6 

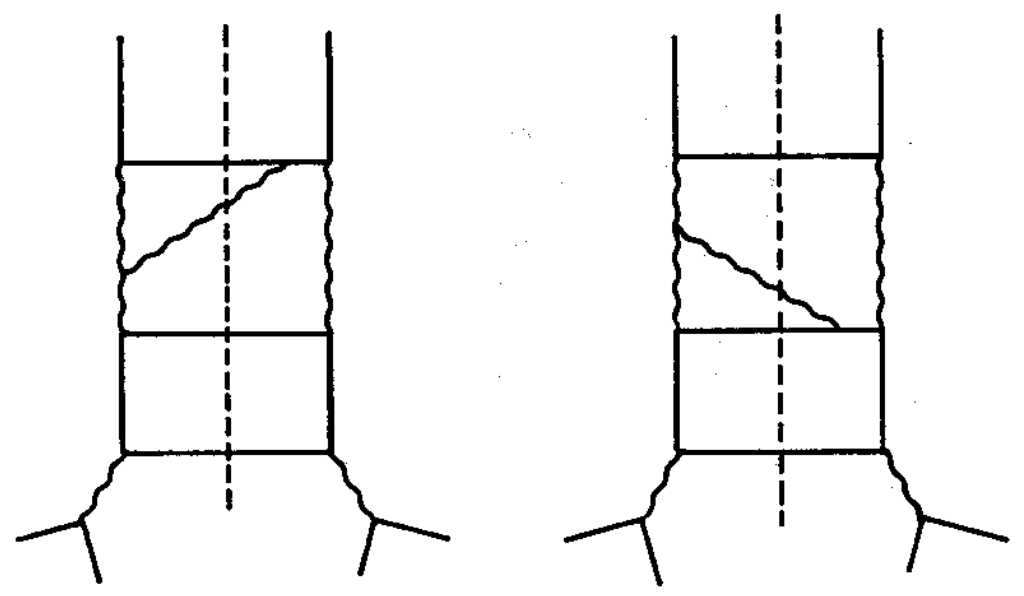

FIG.7
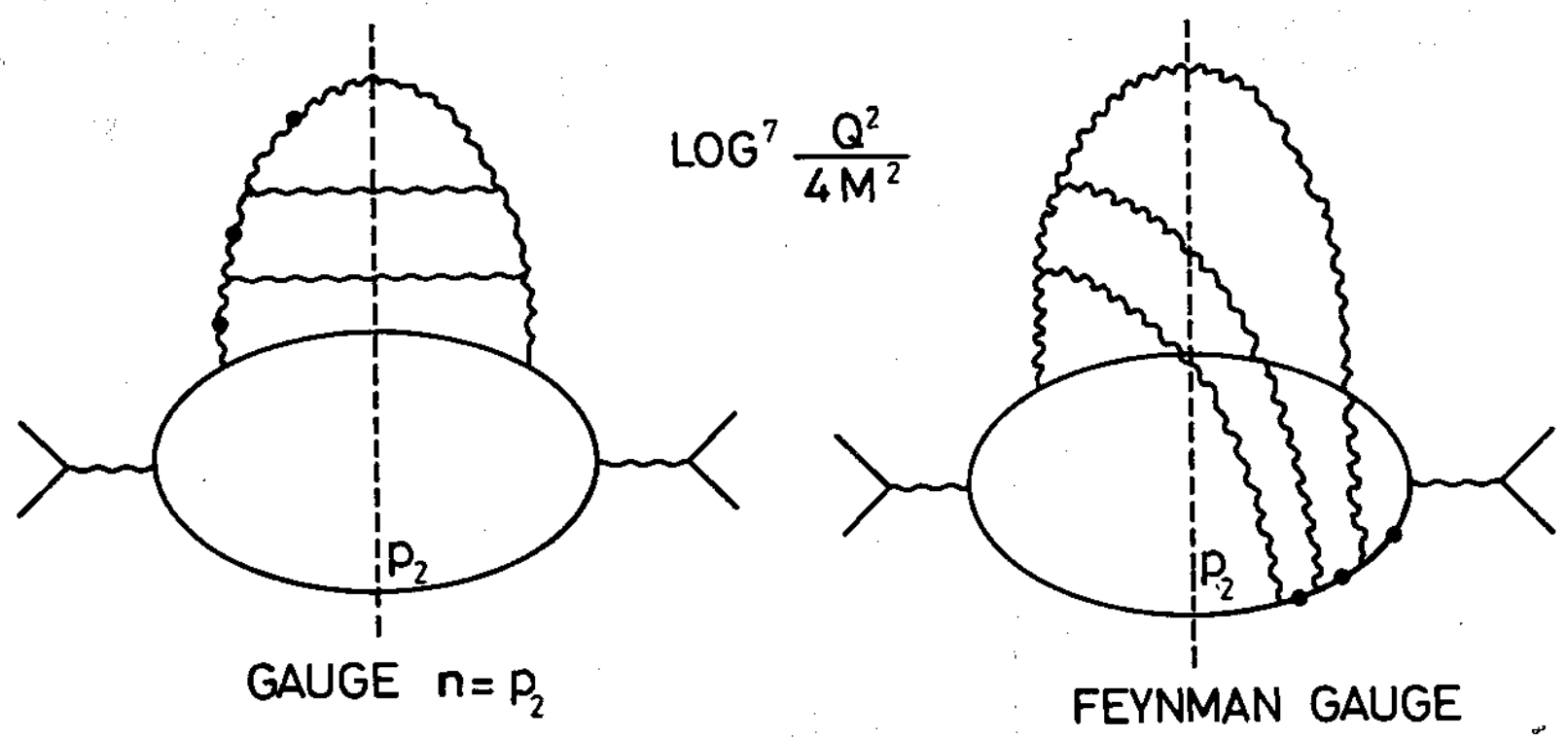

FIG.8 

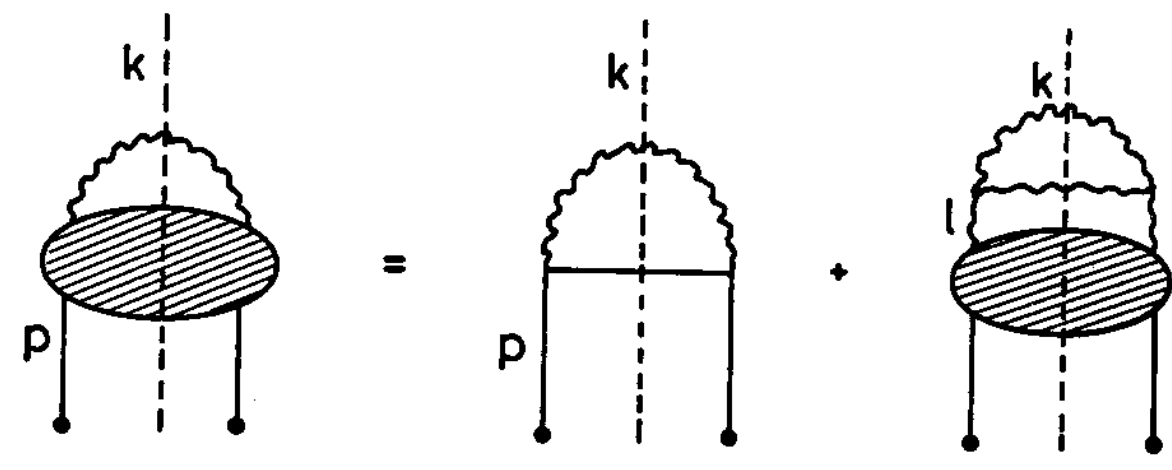

FIG. 9 


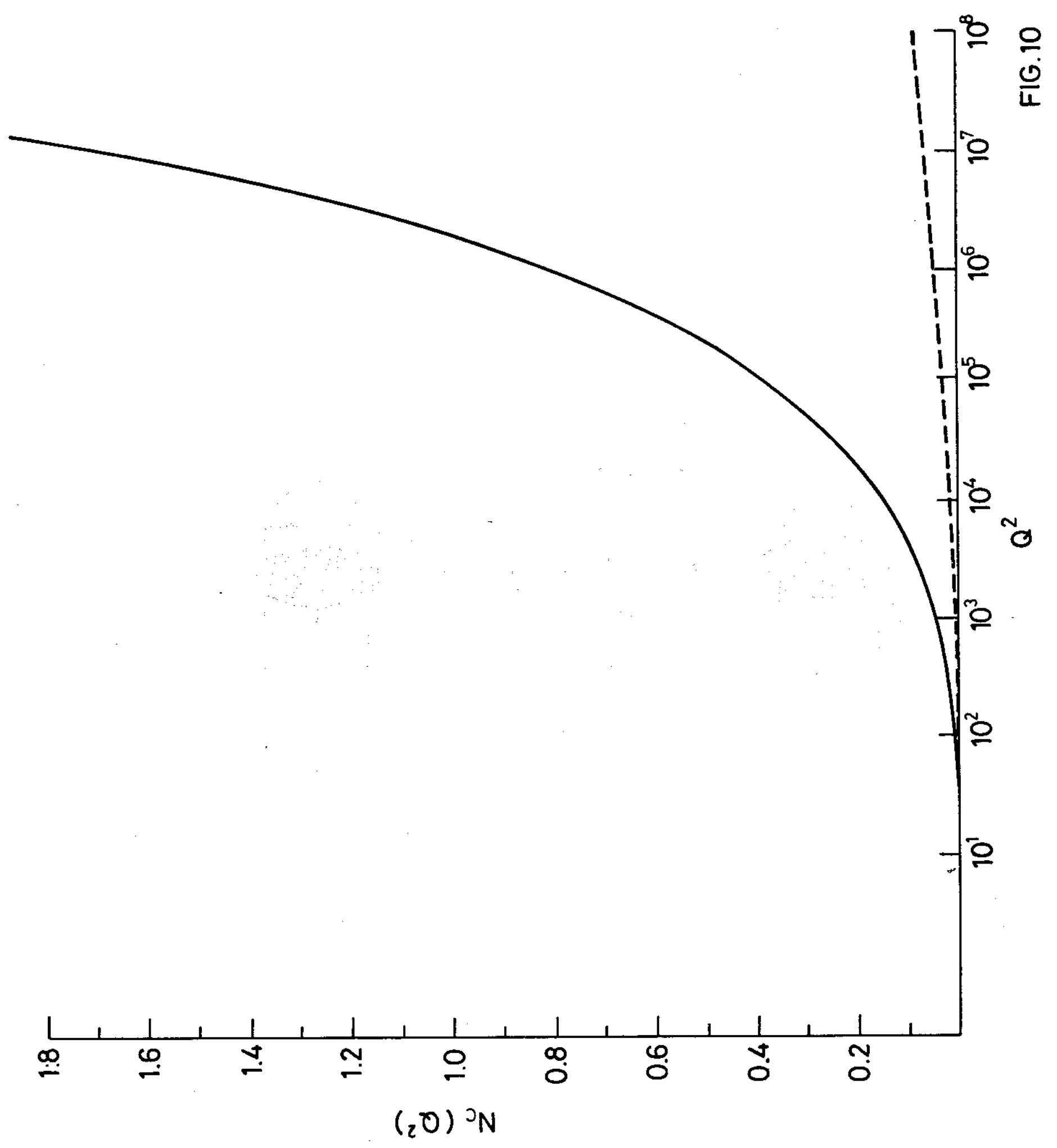

